\title{
Three novel polymorphic microsatellite markers for the glaucoma locus GLC1B by datamining tetranucleotide repeats on chromosome 2p12-q12
}

\author{
Carlos Murga-Zamalloa, Maria Luisa Guevara-Fujita, Alejandro Estrada-Cuzcano and Ricardo Fujita \\ Centro de Genética y Biología Molecular, Facultad de Medicina, Universidad de San Martín de Porres, \\ Lima, Perú.
}

\begin{abstract}
In order to identify new markers around the glaucoma locus GLC1B as a tool to refine its critical region at 2p11.2-2q11.2, we searched the critical region sequence obtained from the UCSC database for tetranucleotide (GATA)n and (GTCT)n repeats of at least 10 units in length. Three out of four potential microsatellite loci were found to be polymorphic, heterozygosity ranging from $64.56 \%$ to $79.59 \%$. The identified markers are useful not only for GLC1B locus but also for the study of other disease loci at 2p11.2-2q11.2, a region with scarcity of microsatellite markers.
\end{abstract}

Key words: GLC1B, microsatellite polymorphic markers, tetranucleotide tandem repeat, gene mapping, glaucoma.

Received: November 12, 2008; Accepted: June 23, 2009.

Primary open angle glaucoma (POAG), an optic neuropathy, is one of the leading causes of blindness (prevalence $3-7 \%$ worldwide), although early detection makes it preventable (Wolfs et al., 2000). POAG heritability is high and family studies revealed at least eight loci with only two causative genes identified so far (Challa, 2004). Locus $G L C 1 B$ was mapped by linkage analysis in British families to a region of about $21 \mathrm{Mb}$ flanked by markers D2S176 and D2S2161 on chromosome 2cen-q13 (Stoilova et al, 1996). We reported a Peruvian family with POAG linked to the $G L C 1 B$ region, with an unaffected member presenting the same haplotype as affected relatives, regarding markers D2S2264, D2S1897and D2S176, but not sharing the alleles at D2S417 and D2S2161 (Fujita et al., 2002 Guevara-Fujita et al., 2003). The segment delimited by D2S176 and $D 2 S 2264$ was discarded for $G L C 1 B$, thus narrowing down the locus critical region to about $15,5 \mathrm{Mb}$ (Fig 1).

In order to refine the location of $G L C 1 B$, we sought markers between D2S2264 and D2S417 which were about $15 \mathrm{Mb}$ apart. However, the reported microsatellite markers did not evenly cover this region, neither were informative in the family we were studying.

This prompted us to search for tetranucleotide repeat markers in the $G L C 1 B$ region, since it is well recognized

Send Correspondence to Ricardo Fujita. Centro de Genética y Biología Molecular, Facultad de Medicina, Universidad de San Martín de Porres, Alameda del Corregidor 1535, La Molina, Lima, Peru. E-mail: rfujita@ rcp.net.pe.

Present addresses: CM-Z, W.K: Kellogg Eye Center, University of Michigan School of Medicine, MI USA; AE-C: Department of Human Genetics, Radboud University Nijmegen Medical Centre, The Netherlands. that tetranucleotide motifs present better allele separation and extra bands than dinucleotide. We searched the NCBI HUMAN GENOME DATASE for these motifs mapping at the segment of about $16 \mathrm{Mb}$ between markers D2S417 and D2S2264 at 2p11.2-2q11.2. Primers flanking candidate markers were designed and their heterozygosity tested in healthy non-related individuals and in our previously reported POAG family. (Fujita 2002, Guevara-Fujita et al, 2003). DNA was obtained from peripheral blood of a total of 106 healthy non-related volunteers using routine salting-out method.

Sequences from 2p11.2 and 2q11.2 (between $D 2 S 417$ and D2S2264) from NCBI BUILD 36.1 available from 'The Human Genome Browser at UCSC' Genomic Library were searched for tetranucleotide repeats with (GATA) n motifs at least 10 units long their flanking sequences, using BIOEDIT SEQUENCE ALIGNMENT EDITOR ${ }^{\circledR}$ software. Selected sequences were analyzed using VECTOR NTI $8^{\circledR}$ (Demo version) for primer design calculations. Reactions were performed in a volume of $10 \mu \mathrm{L}$, containing $50 \mathrm{ng}$ of DNA, $2.5 \mathrm{mM}$ each of dNTPs, $1 \mathrm{mM}$ of each primer, 1 unit of Taq polymerase and $1 \mu \mathrm{L}$ of $10 \mathrm{X}$ buffer with $1 \mu \mathrm{L}$ of $10 \mathrm{X} \mathrm{MgCl}_{2}$. PCR products were amplified on an Amplitron II Thermolyne thermocycler cycling conditions: 35 cycles of $94^{\circ} \mathrm{C}$ for $30 \mathrm{~s}$, optimal annealing temperature for $30 \mathrm{~s}$ and $72{ }^{\circ} \mathrm{C}$ for $30 \mathrm{~s}$. PCR products were electrophoresed on 5 or $6 \%$ denaturing acrylamide gels, alleles subsequently revealed by silver staining. Allele sizes were determined by comparison with a pUC18 sequencing reaction product. 
Four sequences with a (GATA)n motif were identified on the 15,5 Mb segment between 2p11.2 and 2q11.2. To assess heterozygosity, 106 individuals (212 chromosomes) were genotyped from a sample of Lima population, a heterogeneous admixture of South American native and Caucasian ancestries, with minor Asian and African contributions. Three of the identified loci turned out to be polymorphic (Table 1). Figure 1 shows the relative location of the new markers D2SCATTO3, D2SCATTO4, D2SCATTO2 and D2SCATTO1 and of the reference markers between $D 2 S 216$ and $D 2 S 176$ on the GLC1B locus region on chromosome 2 . Table 1 also shows the optimal annealing temperature, allele frequencies, allele size and heterozygosity calculated for each new marker. Marker D2SCATTO1 showed eight alleles ranging from 244 to 272 base pairs (bp) with heterozygosity of $75.15 \%$, marker D2SCATTO2 eight alleles ranging from 319 to $347 \mathrm{bp}$ with heterozygosity of $79.59 \%$, and marker D2SCATTO3 three alleles ranging from 221 to $229 \mathrm{bp}$ with heterozygosity of $64.56 \%$. At D2SCATTO4 only one 291 bp product was obtained in the sample analyzed. Allele sizes of three of the new polymorphic markers allow for multi-loading, making genotyping easier and reducing lab work and time. Primer

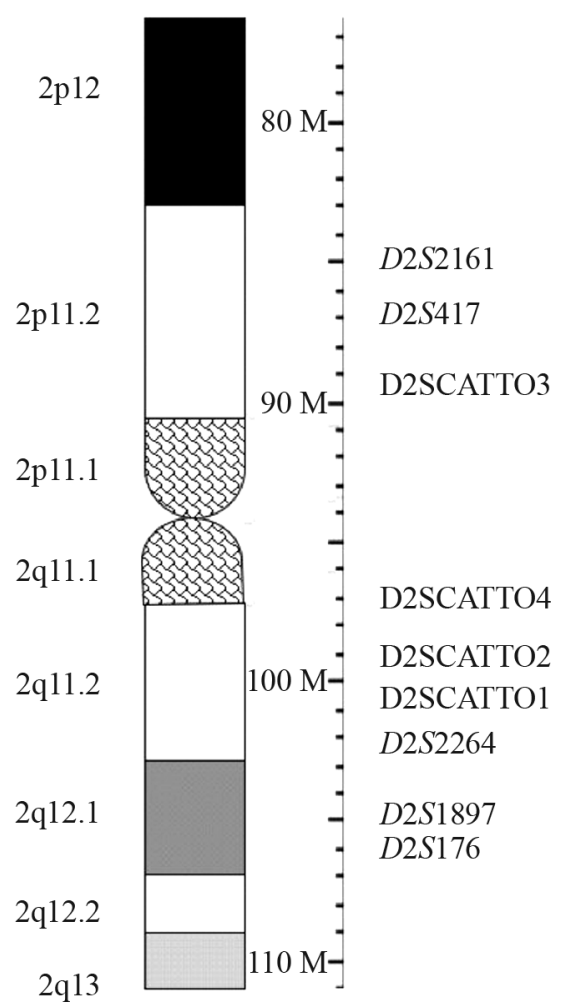

Figure 1 - Cytogenetic localization of 2p11.2-q11.2 markers for Primary Open Angle Glaucoma locus GLC1B [Stoilova et al. (1996) and Fujita et al. (2002)]. The known markers in the region flanked by D2S2161 and D2S176 (D2S417, D2S2264, D2S1897) and generated in this work D2SCATTO3, D2SCATTO4, D2SCATTO2 and D2SCATTO1) are shown. . The ruler the partial map of chromosome 2 shows the relative nucleotide position expressed in Megabases ( $80 \mathrm{Mb}-110 \mathrm{Mb}$, The University of California at Santa Cruz Genome Browser Gateway).

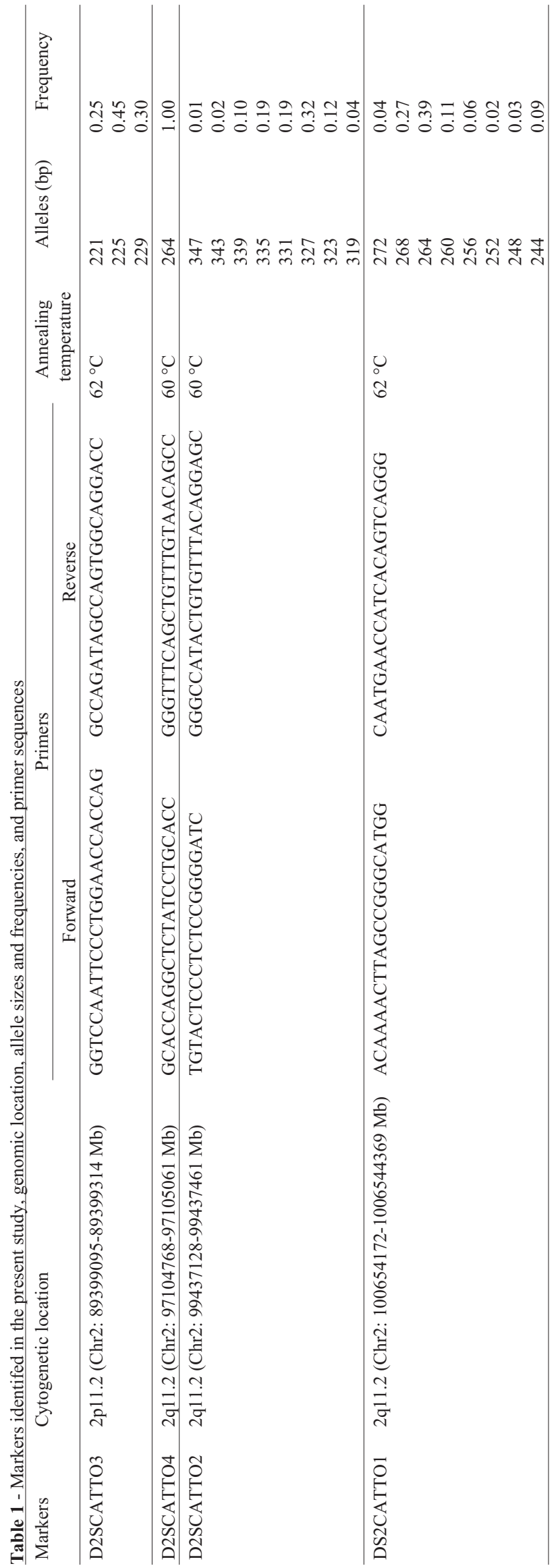


sequences, cytogenetic localization and allele sizes of each marker are deposited in the NCBI dbSNP (BUILD B131, D2SCATTO1 NCBI ss: 142466905 ; D2SCATTO2 NCBI ss: 142466907; D2SCATTO3 NCBI ss: 14246691; 2D2SCATTO4 NCBI ss:142466910).

These markers can be used not only for screening families with POAG populations (Wolfs et al., 2000), but also in other genetic studies in region 2p11.2-q11.2 where loci for different diseases mapped. Examples are Amish Infantile Epilepsy Syndrome (MIM: 609056), Chronic Obstructive Pulmonary Disease with Severe Early-Onset (MIM: 606963), Achromatopsia 2 (MIM: 216900) and Ahnidrotic Ectodermal Dysplasia (MIM: 224900). The study of other diseases mapped the overlapping region such as Schizophrenia (MIM: 181500), Congenital Cataract (MIM: 607304), Combined Deficiency of Vitamin K-Dependent Clotting Factors (MIM: 277450), Ataxia-Telangiectasia (MIM: 208900), Congenital Pulmonary Alveolar Proteinosis (MIM: 178640) Nephronophthisis (MIM: 256100) could also benefit from the use of these markers.

\section{Acknowledgments}

Universidad de San Martin de Porres Funds, Lima, Perú (Project number E20012003005), Consejo Nacional de Ciencia y Tecnología Perú (CONCYTEC).

\section{References}

Challa P (2004) Glaucoma genetics: Advancing new understandings of glaucoma pathogenesis. Int Ophthalmol Clin 44:167-185.

Chung E and Gardiner RM (1996) Mapping human diseases genes by linkage analysis. In: Adolph KW (ed) Methods in Molecular Genetics v. 8: Human Molecular Genetics. Associated Press Inc., San Diego, pp 139-166.
Fujita R, Guevara-Fujita ML, Perez-Grossman R and Richards J (2002) Cosegregation of glaucoma with locus GLC1B (2q12-q13) in a Peruvian family with heterogeneous onset and a recombination within the critical region. Abstracts of the Annual Meeting of The American Society of Human Genetics 71:A1570, pp 439.

Guevara-Fujita ML, Perez-Grossman R, Vargas E and Fujita R (2003) Mapeo cromosómico y refinamiento de la localización de un en de glaucoma en 2q12-q13 en una familia Peruana: Avances hacia la clonación del gen GLC1B. Horizonte Médico 3:28-33 (Abstract in English).

Stoilova D, Child A, Trifan OC, Crick RP, Coakes RL and Sarfarazi M (1996) Localization of a locus (GLC1B) for Adult-Onset Primary Open Angle Glaucoma to the 2cenq13 Region. Genomics 36:142-150.

Wolfs RCW, Borger PH, Ramrattan RS, Klaver CCW, Hulsman CAA, Hofman A, Vingerling JR, Hitchings RA, and de Jong PTVM (2000) Changing views on Open-Angle Glaucoma: Definitions and prevalences - The Rotterdam Study. Invest Ophthalmol Vis Sci 41:3309-3321.

\section{Internet Resources}

NCBI, Human Genome database, www.ncbi.nlm.nih.gov/projects/genome/guide/human/ (September 2004).

The University of California at Santa Cruz Genome Browser Gateway, http://genome.ucsc.edu/cgi-bin/hgGateway (September 2004).

Bioedit. Biological sequence alignment editor for Win95/98/NT/2K/XP http://www.mbio.ncsu.edu/BioEdit/ BioEdit.html. (September 2004).

dbSNP, NCBI, http://www.ncbi.nlm.nih.gov/projects/SNP/ (BUILD B131 August 2009).

OMIM-Online Mendelian Inheritance in Man, www.ncbi. nlm.nih.gov/omim/ (2009).

Associate Editor: Paulo A. Otto

License information: This is an open-access article distributed under the terms of the Creative Commons Attribution License, which permits unrestricted use, distribution, and reproduction in any medium, provided the original work is properly cited. 